\title{
Patientenfreundliche Hochdrucktherapie Bis zur Dreierkombination mit einer Tablette am Tag
}

- Moderne Hypertonietherapie wird heute meistens mit einer Tablette täglich durchgeführt, um eine optimale Compliance zu gewährleisten. Das gilt auch für Kombinationen, die bei den meisten $\mathrm{Pa}$ tienten nötig sind. Nur ein Drittel der Patienten kommt mit einem Wirkstoff aus. Ein weiteres Drittel benötigt zwei Wirkstoffe. Beim dritten Drittel müssen drei und mehr Wirkstoffe kombiniert werden, um den Blutdruck zu kontrollieren, erläuterte Prof. Dr. Carsten Tschöpe von der Charité in Berlin.

Der Notwendigkeit zu kombinieren haben sich auch die europäischen Hypertonie-Leitlinien angenommen: „Die Kombination von zwei antihypertensiven Medikamenten kann bereits in der Initialtherapie vorteilhaft sein", heißt es da, wobei „möglichst Fixkombinationen präferiert werden sollen, weil es die Therapie vereinfacht und die Compliance fördert." "Verschiedene Zweierkombinationen eignen sich für den klinischen Einsatz, doch die meiste Evidenz liegt vor für die Kombinationen RAS-Hemmer plus Diuretika oder RAS-Hemmer plus Kalziumantagonist." Und weiter: „Wenn drei Medikamente benötigt werden, ist die rationalste Kombi-

\section{Abbildung 1}

Blutdruck-Kontrolle im Vergleich

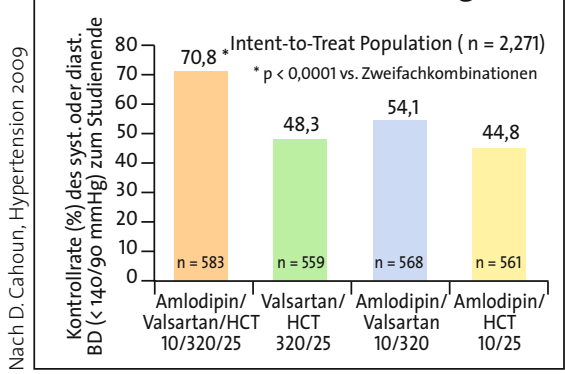

Mit der fixen Dreierkombination erreichen mehr Patienten Werte $<140 / 90 \mathrm{mmHg}$.

nation diejenige aus RAS-Hemmer, Kalziumantagonist und Diuretikum." Eine solche Dreierkombination gibt es als Fixkombination Exforge ${ }^{\circledR}$-HCT. Sie enthält die jeweils meistverordnete Substanz der drei Wirkklassen (Amlodipin, Valsartan, HCT).

\section{Stufenplan für die Behandlung}

Tschöpe empfiehlt, die Hochdrucktherapie in unkomplizierten Fällen mit einem RAS-Blocker zu beginnen. Was in der zweiten Stufe kombiniert wird, hängt vom Patienten ab: Bei Übergewicht oder Diabe- tes zieht er Amlodipin vor, bei Herzschwäche oder Ödemneigung das Diuretikum. Auf Stufe drei erhält der Patient alle drei Wirkstoffe. Auf diese Weise kann der Patient sehr lange mit nur einer Tablette täglich behandelt werden, was die Tablettenlast reduziert und die Therapietreue fördert. Weitere Vorteile: Nur ein Rezept für den Arzt, nur ein Beipackzettel und nur eine Zuzahlung für den Patienten.

Die Wirksamkeit der Dreierkombination ist in einer Studie von D. Cahoun et al. (Hypertension, 2009) untersucht worden. Die Studie lief über acht Wochen mit 2271 Patienten mit einem Ausgangs-RR von $170 / 107 \mathrm{mmHg}$. Verglichen wurde die Dreierkombination mit den drei möglichen Zweierkombinationen. Es zeigte sich, dass mit der Dreierkombination signifikant mehr Patienten den Ziel-RR < 140/90 mm$\mathrm{Hg}$ erreichten (s. Abb.). Gleichzeitig klagten unter der Dreierkombination mit 4,5\% nur ca. halb so viele Patienten über periphere Ödeme wie in den beiden Zweierkombinationen mit Amlodipin.

\section{- Dr. med. Dirk Einecke}

Quelle: Pressegespräch am Rande des AHAJahreskongresses, Orlando, November 2009 (unterstützt von Novartis)

\section{Aufklärungsaktion „Wissen was bei Diabetes zählt: Gesünder unter 7“ Schrittzähler soll Prävention voranbringen}

— Die Diabetes-Aufklärungsaktion „Wissen was bei Diabetes zählt: Gesünder unter 7 " tourt seit fünf Jahren durch deutsche Städte und bietet Tests für das Diabetesrisiko und Aufklärung über die Erkrankung an. Das ist auch nötig, wie die Analyse von 3700 Risikocheck-Fragebögen vom letzten Jahr ergab: Mehr als die Hälfte der Untersuchten hatte ein erhöhtes Erkrankungsrisiko, berichtete Dr. Richard Daikeler, Sinsheim.

Als typische Risiken wurden folgende Faktoren festgestellt: ein erhöhter BodyMass-Index bei $65 \%$, ein erhöhter Taillenumfang bei $48 \%$, eine positive Familien- anamnese für Diabetes bei $45 \%$, zu wenig Bewegung bei $35 \%$, schlechte Ernährungsgewohnheiten bei $16 \%$ und behandelter Bluthochdruck bei $42 \%$. Außerdem lag bei fast jedem Zweiten von 583 Diabetikern der $\mathrm{HbA}_{1 \mathrm{c}}$ über $7 \%$.

\section{Dem Diabetes davonlaufen}

„Natürlich kann man seine Veranlagung nicht ändern“, sagte Prof. Diethelm Tschöpe, Bad Oeynhausen. „Aber man kann wesentlich dazu beitragen, dass ein Typ2-Diabetes sich nicht manifestiert. Dazu gehört an erster Stelle die Bewegung." Bei manifesten Diabetikern kann Bewe- gung die Blutzuckerwerte verbessern und so Folgeschäden vorbeugen. Besucher des Aktionsstandes können in diesem Jahr einen Schrittzähler ausleihen, um ihr Laufpensum zu erfassen - und möglichst auch zu erhöhen. Dass die kleinen Geräte zu mehr Bewegung motivieren, ist in Studien gezeigt worden. Die SchrittzählerAktion wird vom Unternehmen Omron getragen, das innerhalb der Aufklärungsaktion für die Blutdruckkontrolle zuständig ist.

\footnotetext{
- Helga Brettschneider

Quelle: Pressekonferenz, Braunschweig, 18. März 2010 (Veranstalter: Sanofi-Aventis)
} 